\title{
Diversity of rDNA in Chilostomella: Molecular differentiation patterns and putative hermit types
}

\author{
Vera Hemleben ${ }^{1}$; Guido W. Grimm²; Hiroshi Kitazato ${ }^{3} \&$ Christoph Hemleben ${ }^{4}$ \\ ${ }^{1}$ Department of General Genetics, Centre of Plant Molecular Biology (ZMBP), \\ University of Tübingen, Auf der Morgenstelle 28, 72076 Tübingen, Germany \\ vera.hemleben@uni-tuebingen.de \\ ${ }^{2}$ Institute of Geosciences, University of Tübingen, Sigwartstrasse 10, 72076 Tübingen, Germany \\ ${ }^{3}$ Institute for Research and Earth Evolution, Japan Agency for Marine-Earth \\ Science and Technology, Natsushima-cho 2-15, Yokosuta 237-0061, Japan \\ ${ }^{4}$ Institute of Geosciences, Kernlager, University of Tübingen, Sand 6/7, 72076 \\ Tübingen, Germany
}

We screened nuclear DNA sequences coding for the 3' region of the small subunit ribosomal RNA (SSU rDNA), which were obtained from Chilostomella individuals (Foraminifera) for apparent sequence similarities and discrepancies. Sequence characteristics were verified with an enlarged SSU data set and correlated with sequences from the internal transcribed spacer (ITS) regions. Cloning allowed us to evaluate the intraspecific and intraindividual variability. For Chilostomella spec. from various biogeographic areas (North Atlantic, Mediterranean Sea, North Pacific) several highly divergent SSU genotypes were obtained, which could have been misinterpreted as genetically distinct cryptic species or SSU paralogs and produced artificially high intrageneric sequences divergence. Actually, one specific SSU rDNA sequence type (called "domestic" type) was found in most Chilostomella individuals that exhibits the general genotypic characteristics of calcareous test-building foraminifers; this SSU rDNA type showed a convincing biogeographic differentiation pattern, also supported by the ITS data. The other SSU types could be pro parte assigned to agglutinated or monothalamous taxa suggesting contaminants or "hermit" forms associated with the Chilostomella shells. Our findings support the capability of cloning to reveal misleading environmental sequence data and provide, in combination with ITS data, decent insights in population differentiation of benthic foraminifers. In contrast, the benthic foraminifera Virgulinella showed no sequence diversity among the populations from Japan, New Zealand, and Namibia. 